\section{,... vielleicht die Finger davon lassen'}

\section{Zur Fertigstellung der dritten Auflage des ,Schlechter' durch KarLHEINZ SENGHAS}

Dies war das Erste, was der Autor nach Abschluss des Gesamtwerks dem Rezensenten entgegnete, als dieser ihn fragte, was er bei einem zweiten Mal vielleicht anders machen würde. Ganz und gar nicht soll dies aber die Empfehlung an den großen Kreis der an Orchideen interessierten Menschen sein - im Gegenteil: der "SCHLECHTER gehört besonders auch in der dritten Auflage wie schon seit vielen Jahrzehnten in die Handbibliothek jedes ernsthaften ,Orchidisten “ - sei es ein Orchidophiler, ein Orchidologe, ein Orchidomane oder - wie so häufig - von jedem etwas.

Nun ist er also ,fertig - nach vielen Jahren kaum abzuschätzender Arbeit. Begonnen hatte alles lange vor unserer Zeit, als im Jahr 1915 in Berlin die erste Auflage des Werkes unter dem Titel ,Die Orchideen - ihre Beschreibung, Kultur und Züchtung ' erschien. Sie umfasste damals bereits stolze 836 Seite. Sein Autor Rudolf SCHLECHTER (1872-1925) war einer der profiliertesten Orchidologen zumindest des deutschen Sprachraums. Die Verkaufsauflage umfasste 2000 Exemplare, die schon nach wenigen Jahren ausverkauft waren, so dass bereits 1927 eine Neuauflage nötig wurde. Neubearbeitet konnten damals nur die gärtnerisch betonten Teile werden, so dass bereits vor dem zweiten Weltkrieg eine völlige Überarbeitung ins Auge gefasst wurde. Aber erst 1960 konnten mit FrIEDRICH BRIEGER (1900-1985) und RichARD MAATSCH (1904-1992) zwei Verantwortliche für eine dritte Auflage gewonnen werden. Seit 1966 gesellte sich zunächst als Autor, später als Mitherausgeber und in den letzten Jahren als alleiniger Herausgeber KARLHEINZ SENGHAS (geb. 1928) vom Botanischen Garten Heidelberg dazu. So erschien 1970 nach 17-jährigen Vorarbeiten die erste Lieferung der dritten Auflage des ,SCHLECHTER“.

Nach 32 Jahren liegt nun das Werk abgeschlossen vor, wozu dem Autor sowie der Orchideenwelt gratuliert werden kann. Es umfasst zwei Teile, wobei der erste (Botanische Grundlagen und Taxonomischer Teil) allein fast 2900 Seiten in Anspruch nimmt, zu dem sich noch ein Band mit akribisch erstelltem Register und einem umfangreichen Literaturverzeichnis von noch einmal 268 Seiten gesellt. Der 1985 publizierte zweite Teil (Erwerbsgartenbau, Pflege im Zimmer, Freilandorchideen, Krankheiten und Naturschutz) bringt es noch einmal auf 727 Seiten. Insgesamt umfasst der ,SCHLECHTER' fast 4000 Seiten Orchideenkunde, gebunden in vier Bänden. Die Feststellung, das gesamte Wissen unserer Zeit über Orchideen sei hier versammelt, ist bestimmt nur eine kleine Übertreibung und eher eine Berücksichtigung der Tatsache, dass all unser Tun als Menschen immer nur ein fragmentarisches sein kann.

So werden allein in dem ersten Teil des Werkes 12000
Orchideennamen behandelt, etwa 2000 Titel von Arbeiten zur Orchideenkunde aus den Jahren zwischen 1752 und 2002 sind im Literaturverzeichnis zusammengestellt, dazu gibt es eine Auflistung der Orchideenzeitschriften und der für die Orchidologie besonders wichtigen Zeitschriften. Die letzte Abbildung zeigt auf S. 2823 mit ihrer Nummer 2819, dass statistisch fast auf jeder Seite ein Bild anzutreffen ist. Besonders erwähnenswert ist die Aktualität des Werkes, dessen erste Lieferung immerhin vor 32 Jahren erschienen ist: durch umfangreiche Nachträge immerhin fast 400 Seiten - ist es auf dem ,aktuellstmöglichen' Stand. Denn es konnten für den Druck noch bis Ende 2001 erschienene Arbeiten berücksichtigt werden.

Als Besonderheit des ,SCHLECHTER' ist der Bezug zu den kultivierten Arten hervorzuheben, die das Werk weit über die engere Wissenschaftsgemeinde der Orchidologen hinaus für den Praktiker nahezu unverzichtbar macht. Wichtige und interessante Informationen bietet das Werk für Gärtner, Liebhaber oder Institutionen wie Botanische Gärten oder auch Behörden im Artenschutzbereich. Das einzige Hemmnis hierfür ist natürlich der Preis; wo wir Subskribenten maximal drei Jahrzehnte ,abstotterten', schlägt der Gesamterwerb der immerhin über, $10 \mathrm{~kg} \mathrm{Buch}$ “ mit etwa 940,- $€$ zu Buche bzw. eine große Lücke in die Finanzplanung.

Den Bezug zur Orchideenkultur verdankt das Werk einerseits der Lebendsammlung eines Botanischen Gartens und den dort versammelten gärtnerischen Kenntnissen und Fähigkeiten sowie dem Kontakt zu vielen Liebhabern, auf deren Erfahrungen ebenfalls zurückgegriffen werden konnte. So sind die Bände auf allen Ebenen (bis hin zu einzelnen Arten!) vollgepackt mit breitestem biologischen Wissen - Morphologie, Taxonomie, Systematik, Ökologie und praktische Hortikultur. Auch ein großer Teil der Abbildungen verdankt ihre besondere Qualität der Tatsache, dass lebendes Material als Vorlage zur Verfügung stand.

An dieser Stelle ist besonders dem Verlag Dank auszusprechen, der in den wechselnden Zeitläufen und bei zweimaligem Wechsel dem Werk die Treue gehalten und es zu einem guten Ende gebracht hat. Gedankt sei an dieser Stelle auch dem Dienstherren des Herausgebers, der Universität Heidelberg, dem dortigen Botanischen Institut und dem Botanischen Garten: erst durch deren ,Langmut' (ganz im Gegensatz zum Zeitgeist, der eher dazu neigt, die schiere Anzahl der jährlichen Publikationen zum Maß der Dinge zu machen) war es möglich, über die Jahre hinweg ein solches Werk reifen und entstehen $\mathrm{zu}$ lassen.

Dies ist einer der Gründe, warum diese Auflage des 'SCHLECHTER' sicherlich das letzte Werk dieser Größenordnung gewesen sein wird, das zum großen Teil von einem Einzelnen bearbeitet und verfasst worden ist. Angesichts der administrativen Hemmnisse wie CITES oder ähnlicher Konventionen wird es in Zukunft kaum noch 
möglich sein, eine so umfangreiche Lebendsammlung zusammenzutragen, wie dies in Heidelberg geschehen ist dies umso mehr im Angesicht der Tatsache dass es eine Reihe von Ländern gibt, die die Ausfuhr lebenden biologischen Materials grundsätzlich kaum mehr gestatten.

Und zum Schluss natürlich vom Herzen ein Dank der ,Orchideengemeinde' und des Rezensenten an KARLHEINZ SENGHAS. Der Rezensent ist seit 1987 Subskribent des Werkes und hat wie viele andere stets die neue Lieferung gespannt erwartet und sich mit den wildesten ,Privatregistern' beholfend - auch über diese Not ließe sich einmal trefflich schreiben. Ein großes Lob sei KARLHEINZ SENGHAS dafür ausgesprochen, dass er eben nicht die Finger davon gelassen hat und treu über viele Jahre hinweg diese ,Kärrnerarbeit“ immer wieder geschultert, geleistet und zu einem bravourösen Abschluss gebracht hat. Daneben ist dem Werk eine weite Verbreitung und vielleicht auch die Weiterentwicklung hin auf eine englische Übersetzung oder seine elektronische Verfügbarmachung zu wünschen. Wir Nutzer jedenfalls sollten beim In-die-Hand-Nehmen eines der Bände immer wieder einmal dankbar des Herausgebers gedenken.

\section{SCHLECHTER, R. (Begr.)}

Die Orchideen

Beschreibung, Kultur und Züchtung

Herausgegeben von F. G. BRIEGER, R. MAATSCH \&

K. Senghas.

Zwei Teile und ein Registerband, gebunden in vier Bänden (Teil 1 in drei Bänden, Teil 2 in einem Band, ein Registerband), Verlag Paul Parey.

Preis des Gesamtwerks: ca. 940,- $€$.

\section{Gärtnerisch-botanische Literatur}

\section{Alma De L'Aigle}

\section{Ein Garten}

Dölling und Galitz Verlag, Hamburg, 1996, 182 Seiten, 25 Farbabbildungen, geb. 19,80 €; ISBN 3-930802-39-2.

Dass der Besitz oder zumindest die Nutzungsmöglichkeit eines Gartens Menschen zum Bessern bekehren kann, möchte man nach der Lektüre von AlMA DE L'Aigles Buch „Ein Garten“ glauben.

Die Wiederentdeckung und Neuauflage dieses Buches sind ein wirklicher Glücksfall für alle Gartenliebhaber. Zudem konnte in diesem Zuge ein Stück des beschriebenen Paradieses in Hamburg gerettet werden. Es ist unglaublich, wie viele Adjektive Alma DE L'Aigle für Form, Farbe, Duft und Geschmack von Obst und Gemüse, aber auch für Bäume und Blumen, findet. „Außer der Linde, die einen oft geradezu überschüttet mit ihrem starken Duft, weiß ich nur eine Blüte, die sich mit der Weinblüte an Zartheit, Lieblichkeit und Reichtum des Duftes messen kann: die ebenso unscheinbare Blüte der Stachelbeere, zu der man sich niederbeugen muss wie zum Veilchen, um ihre Gabe zu empfangen.“

Bereits in diesem kurzen Abschnitt meint man, dass der beschriebene Duft den Seiten des Buches entströmt. Ich habe dieses Buch im Winter gelesen und hatte den Eindruck, dass es nun schneller Frühling werden würde. Ganz nebenbei erfährt der Leser, welche Pflanzen lieber im Schatten stehen oder die pralle Sonne genießen, welche Nachbarschaften sie lieben oder ob sie feuchte oder trockene Standorte bevorzugen. Die Autorin beschreibt mit gleicher Leidenschaft Rosen und Kamelien wie Löwenzahn, Breitwegerich oder Schafgarbe. Die Beschreibung von Blättern und Wuchsformen sowie der verschiedensten Grüntöne lesen sich wie Kunstbetrachtungen und vermitteln gleichzeitig viel gärtnerisches Wissen.

Man bekommt einfach gute Laune, wenn man Alma de l'Aigles Beschreibung des Gartens ihrer Eltern in Hamburg Lokstedt liest. Das Obst, das ihr Vater kultivierte, wurde zum Teil in den besten Delikatessenläden Hamburgs verkauft. Er hatte sich als Jurist mit einer halben Stelle begnügt, um in seinem Garten sein zu können, weshalb der Garten auch ein wenig zum familiären Einkommen beitragen musste.

„Ja, Gärten und Kinder sind es, um die es sich lohnt zu leben. Ihnen den Bezug zur Mitte, den Duft der Seele, die Echtheit zu erhalten war und bleibt mein Lebensziel." So endet das Buch von AlmA DE L'Aigle, die von 1889-1959 gelebt hat. In diesem kurzen Satz ist viel über ihre Person gesagt. Eigentlich wollte sie Malerin werden. Bei ihrer nachweislichen Beobachtungsgabe und den Bildern von ihr im Buch wäre das sogar vielversprechend gewesen. Aber sie wurde Lehrerin und hat sich vor allem für die Bildung der benachteiligten Kinder eingesetzt. Sie war eine leidenschaftliche Reformpädagogin, die viele ihrer Schüler mit in den elterlichen Garten genommen hat.

Ihre besondere Liebe galt den Rosen. So empfehle ich allen auch ihr zweites Buch: „Begegnung mit Rosen“, das im gleichen Verlag erschienen ist.

KARIN WITTSTOCK 